\title{
Ion-beam induced nano-sized Ag-metal clusters in glass
}

\author{
H.-E. Mahnke ${ }^{\mathrm{a}, \mathrm{b}, *}$, I. Zizak ${ }^{\mathrm{a}, 1}$, P. Schubert-Bischoff ${ }^{\mathrm{a}}$, V. Koteski ${ }^{\text {a,c }}$ \\ ${ }^{a}$ Hahn-Meitner-Institut Berlin GmbH, Bereich SF, Glienicker Str. 100, D-14109 Berlin, Germany \\ ${ }^{\mathrm{b}}$ FU Berlin, D-14195 Berlin, Germany \\ ${ }^{\mathrm{c}}$ VINČA, POB 522, 11001 Belgrade, Serbia
}

Received 22 October 2007; received in revised form 23 November 2007; accepted 27 November 2007

\begin{abstract}
The formation of silver-metal clusters has been studied in ion-exchanged soda-lime glass following swift heavy ion irradiation at ISL. While the transformation into a metallic chemical form was detected with X-ray absorption spectroscopy (EXAFS) the shape and geometrical arrangement was studied both by transmission electron microscopy (TEM) and small angle X-ray scattering (SAXS). A chain-like arrangement of nanoclusters of approximately $7 \mathrm{~nm}$ in diameter observed in TEM was further investigated with in situ SAXS during the subsequent annealing process. (c) 2007 Elsevier B.V. All rights reserved.
\end{abstract}

Keywords: Metallic nanoclusters in glass; Small angle X-ray scattering (SAXS); Ion beam modification; Ion tracks; Transmission electron microscopy (TEM); $\mathrm{X}$-ray absorption spectroscopy (XAS)

\section{Introduction}

Glasses containing transition metal clusters have attracted attention both in cluster research and in possible applications of such clusters for magnetic or optoelectronic purposes. Nanometer-sized clusters of the noble metals $\mathrm{Au}$ and $\mathrm{Ag}$ in glasses exhibit strong absorption in the visible spectral range. The light absorption, in addition, may be highly sensitive to the polarization depending on size and shape of the clusters $[1,2]$. Special arrangements of the nanoclusters, their alignment in particular, may be used for waveguiding as already discussed and studied (e.g. Refs. [3,4]).

The new possible applications of such metallic nanoclusters have led to a renewed interest in investigating the binary exchange technique for introducing the respective metals into the glass, already used since centuries in making colourful glasses. Various steps must be understood for a better control: the introduction of the metal atoms into the glass which typically results in a non-metallic chemical environment (step i), the transformation into metal (step ii) and cluster formation with a control of size, shape, and arrangement (step iii). It is obvious that these steps are interrelated and interdependent. They are strongly

\footnotetext{
* Corresponding author.

E-mail address: mahnke@hmi.de (H.-E. Mahnke).

1 Now at BESSY GmbH, D-12489 Berlin, Germany.
}

influenced by the presence of reducing constituents or impurities in the glass. The means by which the energy is brought in, needed for reducing the elements typically incorporated in the glass in oxidic environment to metal, are, besides heat (annealing) treatment, laser (light) or ion irradiation. Studies using laser irradiation and irradiations with light or low energy ions with only little energy transfer to the electrons in the material ("electronic stopping") combined with annealing procedures already showed some influence on the cluster formation and size distribution $[5,6]$. In the most recent studies using swift heavy ions, a "directionality" showed up as a special feature of the metallic cluster formation in glass: metallic silver clusters were observed in a chain-like arrangement along the direction of the ion beam $[7,8]$. This finding has to be attributed to the higher energy density being transferred to the electronic system of the material which seems to be sufficiently high enough above the threshold for ion track formation in these glasses in the case of irradiation with Si ions used in Ref. [7] and well above the threshold in the case of the Au ion irradiation used in our previous [8] and present investigation.

Starting from soda-lime glass into which silver was introduced by ion exchange, Ag-metal clusters were observed in transmission electron microscopy (TEM) images which were induced by heavy ion irradiation and subsequent annealing under reducing atmosphere. The transformation of Ag from the oxidic chemical configuration after the ion-exchange process into the metallic form was detected with X-ray absorption spectroscopy 